\title{
Low-temperature behavior of the ESR linewidth in a system with a spin gap: $\eta-\mathrm{Na}_{1.286} \mathrm{~V}_{2} \mathrm{O}_{5}$
}

\author{
F. Chabre, ${ }^{1}$ A. M. Ghorayeb, ${ }^{1}$ P. Millet, ${ }^{2}$ V. A. Pashchenko, ${ }^{3}$ and A. Stepanov ${ }^{1}$ \\ ${ }^{1}$ Laboratoire Matériaux et Microélectronique de Provence, CNRS UMR 6137, Case 142, Faculté des Sciences de St-Jérôme, \\ Université d'Aix-Marseille III, 13397 Marseille Cedex 20, France \\ ${ }^{2}$ Centre d'Elaboration de Matériaux et d'Etudes Structurales, CNRS UPR 8011, B.P. 4347, 29 rue Jeanne Marvig, \\ 31055 Toulouse Cedex, France \\ ${ }^{3}$ Physikalisches Institut, Johann Wolfgang Goéthe Universität, Robert-Mayer-Strasse 2-4, 60054 Frankfurt am Main, Germany
} (Received 9 August 2004; revised manuscript received 13 April 2005; published 21 July 2005)

\begin{abstract}
Using X-band electron spin resonance (ESR) spectroscopy down to $4.2 \mathrm{~K}$, we report on the low-temperature magnetic behavior of $\eta-\mathrm{Na}_{1.286} \mathrm{~V}_{2} \mathrm{O}_{5}$, a system with a spin gap. We particularly find that the ESR linewidth of this compound shows an unusual temperature dependence, rich in features. Addressing the problem via the memory function formalism, we propose an explanation for this, whereby we suggest that this unconventional low-temperature behavior is related to the way in which the magnetic susceptibility and the memory function depend on temperature, rather than to a specific magnetic origin.
\end{abstract}

DOI: 10.1103/PhysRevB.72.012415

PACS number(s): 76.30.-v, 75.50.Ee, 76.50.+g

The electron spin resonance (ESR) technique, owing to its high sensitivity and resolution, has proven to be a very powerful tool for studying the magnetic interactions in many low-dimensional systems which are of interest at present, such as, for instance, vanadates and cuprates (see, e.g., Refs. 1-4 and references cited therein). In particular, the linewidth of the ESR spectra provides important information concerning the spin dynamics within the system. However, and in the case of spin- $\frac{1}{2}$ Heisenberg antiferromagnets (AFM) in particular, the lack of a proper ESR theory has for a long time hindered the interpretation of many experimental studies. The main reason for this is the difficulty in calculating what is generally called the self energy or the memory function which appears in the expression of the ESR linewidth. ${ }^{5-7}$ Recently, Oshikawa and Affleck, ${ }^{8}$ using the field theory approach, have performed an extensive theoretical study of the electron spin resonance in $S=\frac{1}{2}$ antiferromagnetic chains. However, their study did not cover the case of spin-gap systems.

Our work in this report focuses, through an ESR study of the spin-gap system $\eta-\mathrm{Na}_{1.286} \mathrm{~V}_{2} \mathrm{O}_{5}$, on determining the temperature dependence of the memory function experimentally, in order to provide an interpretation for the behavior of the linewidth which, in this compound, shows a particularly unusual temperature dependence. Our analysis leads us to the conclusion that the unconventional behavior of the linewidth in this system does not represent peculiar variations in spin dynamics, but simply results from the way in which the magnetic susceptibility and the memory function depend on temperature.

The vanadium oxide bronze $\eta-\mathrm{Na}_{1.286} \mathrm{~V}_{2} \mathrm{O}_{5}$, which can also be denoted by the stoichiometric formula $\mathrm{Na}_{9} \mathrm{~V}_{14} \mathrm{O}_{35}$, crystallizes in the monoclinic system (space group $P 2 / c$ ) with a structure built up of layers of $\mathrm{VO}_{5}$ square pyramids sharing edges and corners, and having their apical oxygen pointing up and down alternately. ${ }^{9,10}$ These pyramids form double strings in the [100] direction, which are separated, in the [001] direction, by $\mathrm{VO}_{4}$ tetrahedra. A particular aspect of these double strings, however, is that they show a stair-like shape with a step every ten $\mathrm{VO}_{5}$ square pyramids. The $\mathrm{Na}$ atoms are distributed between the layers. At room temperature, the two vanadium ions lying at the extremities of the ten-pyramid segment (i.e., those which ensure the link with the neighboring segments) are in the $\mathrm{V}^{4.5+}$ state, whereas the remaining eight vanadium ions of each segment are in the $\mathrm{V}^{4+}$ state. ${ }^{9,10}$ Hence each segment contains nine spins $\frac{1}{2}$. On the other hand, the magnetic susceptibility of this compound ${ }^{10}$ shows, as mentioned above, a spin gap behavior which, at first sight, is incompatible with the case of a system containing an odd number of spins. A tentative explanation for the origin of the spin gap was then given by Whangbo and Koo, ${ }^{11}$ who proposed, using a spin dimer analysis, that the $\mathrm{V}_{10} \mathrm{O}_{30}$ chains of this compound are topologically equivalent to a fused chain of 10-node rings. This interpretation, however, remains hard to reconcile with the fact that each magnetic unit contains, as mentioned above, nine spins $\frac{1}{2}$. Very recently, low-temperature structural measurements ${ }^{12}$ have revealed the existence, around $100 \mathrm{~K}$, of a structural second-order phase transition in this compound, associated with a concomitant charge ordering. The low-temperature structure remains centrosymmetric with space group $P 2 / c$ but with a doubled $b$ lattice parameter in comparison with that at high temperature, and the two vanadium sites which host $\mathrm{V}^{4.5+}$ ions at room temperature become stabilized as one $\mathrm{V}^{4+}$ and one $\mathrm{V}^{5+}$ at low temperature. The dimerization along the $\boldsymbol{b}$ axis observed below $100 \mathrm{~K}$ means that each magnetic unit contains, at low temperature, $2 \times 9$ spins $\frac{1}{2}$. The origin of the spin gap can hence be related to the formation of this superstructure below $100 \mathrm{~K}$ in the system. Preliminary $\mathrm{X}$-band electron-spin resonance (ESR) and complementary magnetic susceptibility data, which we reported recently, ${ }^{12}$ give additional evidence for the transition around $100 \mathrm{~K}$ and confirm the existence of the spin gap, which we have estimated to be of the order of $35 \mathrm{~K}$.

Concerning the study which we are reporting here, single crystals of $\eta$ - $\mathrm{Na}_{1.286} \mathrm{~V}_{2} \mathrm{O}_{5}$ were prepared following the same procedure as described in Ref. 12, and studied by ESR spectroscopy using a Bruker EMX spectrometer operating in the 


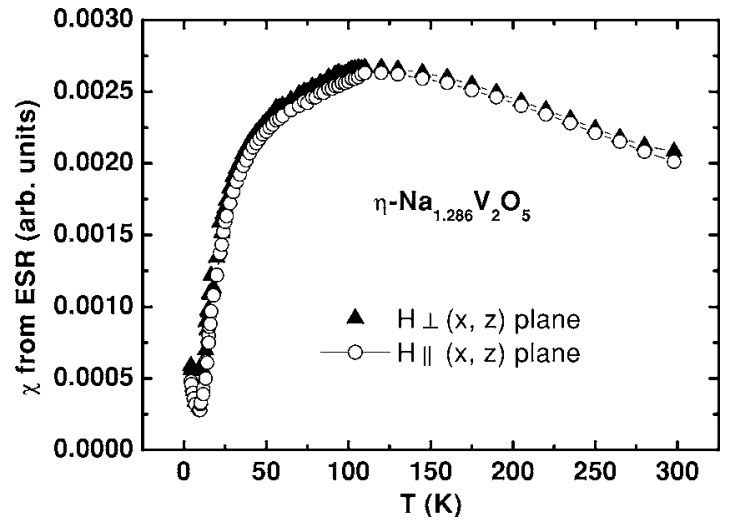

FIG. 1. Temperature dependence of the susceptibility of $\eta$ $\mathrm{Na}_{1.286} \mathrm{~V}_{2} \mathrm{O}_{5}$ as deduced from the ESR spectra. Triangles and circles correspond to data obtained for $\boldsymbol{H}$ perpendicular and $\boldsymbol{H}$ parallel to the $(x, z)$ plane, respectively.

X-band $(\nu=9.635 \mathrm{GHz})$. The static magnetic field, $\boldsymbol{H}$, was applied in both directions, parallel and perpendicular to the plane containing the double strings and to which we shall refer as the $(x, z)$ plane of the structure, following the notation of Ref. 12.

The obtained room-temperature $g$-factor values, $g_{\|}$ $=1.973$ and $g_{\perp}=1.931$, are typical of $\mathrm{V}^{4+}$ ions in a fivefold pyramidal environment (see, e.g., Refs. 13 and 14).

Figure 1 shows the temperature dependence of the magnetic susceptibility of a typical sample of $\eta-\mathrm{Na}_{1.286} \mathrm{~V}_{2} \mathrm{O}_{5}$, as deduced from the ESR spectra. Data obtained for $\boldsymbol{H}$ perpendicular (triangles) and $\boldsymbol{H}$ parallel (circles) to the $(x, z)$ plane are shown. The close similarity between the two curves indicates that the magnetic interactions may be regarded as nearly isotropic. The temperature dependence of the spin susceptibility shown in Fig. 1 is similar to that measured by SQUID magnetometry and which we reported in Ref. 12: in particular, we note the small kink just above $100 \mathrm{~K}$ associated with the occurrence of the second-order phase transition mentioned above, as well as the decrease towards zero below this temperature indicating the presence of the spin gap (the low-temperature increase below $7 \mathrm{~K}$ is attributed to impurities, as we explained in Ref. 12).

The temperature dependence of the ESR linewidth, $\Delta H_{1 / 2}$ (half width at half-maximum), is shown in Fig. 2, for both field directions, $\boldsymbol{H}$ perpendicular (filled circles) and $\boldsymbol{H}$ parallel (open circles) to the $(x, z)$ plane. We may clearly see in this figure that, although the linewidth is anisotropic, its temperature dependence is almost independent of the field direction (apart from the $80 \mathrm{~K}$ peak which appears in the case where $\boldsymbol{H}$ is perpendicular to the $(x, z)$ plane and on which we shall comment below). If we closely examine the temperature behavior of this linewidth, we may distinguish four different regimes.

(i) For temperatures $T \gtrsim 230 \mathrm{~K}$, a slow increase of the linewidth is noted with increasing $T$, as generally expected at these temperatures where the linewidth gently increases before reaching saturation at higher temperatures owing to the disappearance of the magnetic correlations.

(ii) In the temperature range $30 \mathrm{~K} \lesssim T \leqq 230 \mathrm{~K}$, the linewidth shows a peak at around $80 \mathrm{~K}$ when $\boldsymbol{H}$ is perpendicular

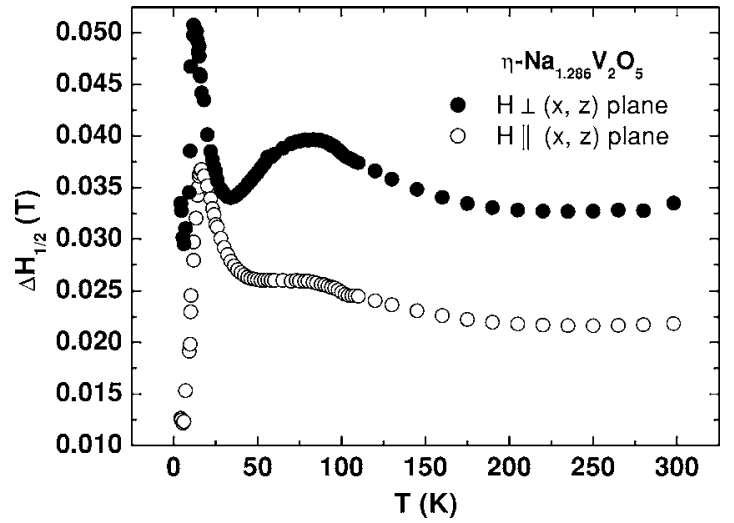

FIG. 2. Temperature dependence of the ESR linewidth, $\Delta H_{1 / 2}$, of $\eta-\mathrm{Na}_{1.286} \mathrm{~V}_{2} \mathrm{O}_{5}$. Filled and open circles correspond to data obtained for $\boldsymbol{H}$ perpendicular and $\boldsymbol{H}$ parallel to the $(x, z)$ plane, respectively.

to the $(x, z)$ plane, while this peak is suppressed when $\boldsymbol{H}$ is parallel to the $(x, z)$ plane. Furthermore, in both field directions, a kink appears at around $100 \mathrm{~K}$ in the curves. We attribute this behavior to the magnetic correlations accompanying the second-order phase transition reported in Ref. 12 and which we mentioned above. The discrepancy between the two curves as regards the presence or the absence of the $80 \mathrm{~K}$ peak would then be directly related to the fact that the phase transition consists basically of a dimerization along the $y$ direction of the structure.

(iii) For $17 \mathrm{~K} \lesssim T \lesssim 30 \mathrm{~K}$, a rapid increase of the linewidth is observed with decreasing temperature. Such a rapid increase may usually be regarded as the manifestation of critical antiferromagnetic fluctuations. ${ }^{15-17}$ In that case, the temperature dependence of the linewidth follows a power law, the critical exponent of which being related to the dimensionality of the system. We have tried to fit $\Delta H_{1 / 2}$ to such a law in the corresponding temperature range, but no such dependence could unambiguously be obtained. The explanation for the behavior of $\Delta H_{1 / 2}$ in this regime remains thus unclear at this stage but will be clarified below.

(iv) For $T \lesssim 16 \mathrm{~K}$, a very rapid decrease of the linewidth is noted as the temperature decreases. Such a decrease of the linewidth at low temperatures has already been observed by Taniguchi et al. ${ }^{18}$ in their study of the quasi-two-dimensional (2D) spin-gap system $\mathrm{CaV}_{4} \mathrm{O}_{9}$, but no clear interpretation of this was then given. In fact, this type of behavior is quite different from what is generally observed at low temperature in other spin-gap systems such as, for instance, $\alpha^{\prime}-\mathrm{NaV}_{2} \mathrm{O}_{5}$ or $\mathrm{CuGeO}_{3}$ (see, e.g., Refs. 1-3), in both of which the linewidth presents a minimum at low temperature. We believe that the type of behavior which we observe here in the case of $\eta-\mathrm{Na}_{1.286} \mathrm{~V}_{2} \mathrm{O}_{5}$, albeit rare, is what would normally be expected in a system with a spin gap.

For a more quantitative discussion of the dynamic spinspin correlations, one has to consider the memory function. Indeed, the ESR linewidth in the exchange-narrowing regime may be expressed as ${ }^{7,19}$ 


$$
\gamma \Delta H=\frac{\int_{-\infty}^{+\infty} F(\tau) d \tau}{8 \hbar^{2}\left\langle M_{x}^{2}\right\rangle},
$$

where $\gamma$ is the gyromagnetic ratio, $\Delta H$ is the full linewidth and $M_{x}$ is the total $x$ component of magnetization. The numerator of expression (1), which we shall call $F$, is a sum of four-spin correlation functions called the memory function. Generally, $F$ is difficult to calculate but, in the case where

$$
\gamma \Delta H \ll \omega_{0},
$$

(where $\omega_{0}$ is the resonance angular frequency), $F$ can be approximated by ${ }^{19}$

$$
\int_{-\infty}^{+\infty} F(\tau) d \tau \propto F=\chi_{0} \cdot \Delta H \cdot \operatorname{coth} \frac{\hbar \omega_{0}}{2 k_{B} T},
$$

where $\chi_{0}$ is the area under the absorption curve and hence represents the static susceptibility. In our case, condition (2) is satisfied since $\gamma \Delta H$ is an order of magnitude smaller than $\omega_{0}$. Therefore, the memory function, $F$, becomes here a measurable quantity, proportional to the product that appears in the second part of expression (3). In the case where $\hbar \omega_{0}$ $\ll k_{B} T$ (which is satisfied here), this product can be further approximated to

$$
F \propto \chi_{0} \cdot \Delta H \cdot T .
$$

Let us now turn to the linewidth temperature behavior. As it follows from the above formula, $\Delta H(T)$ is proportional to the memory function, $F(T)$, divided by $\chi_{0}(T) \cdot T$. This means that for a system where the static susceptibility is finite at low temperature, $\Delta H(T)$ simply reproduces the ratio $F(T) / T$. [For example, in the case of a $S=\frac{1}{2}$ AFM Heisenberg chain, $F(T) \sim T^{2}$ and $\Delta H(T)$ scales as $T$ (Refs. 8, 20).] In the case of gapped systems, the behavior of $\Delta H(T)$ is more complicated. Here one would expect that both static and dynamic correlation functions, due to the depopulation of the excited states as the temperature is lowered, will have a tendency to rapidly decrease. Then the linewidth is essentially a ratio of two dropping-to-zero functions, thus resulting in nonmonotonic behavior (singularities) of $\Delta H(T)$. This is just the case of $\eta-\mathrm{Na}_{1.286} \mathrm{~V}_{2} \mathrm{O}_{5}$.

We have plotted in Fig. 3, not only the temperature dependence of $F$ (which appears in the inset), but also the temperature dependence of the ratio $F / T$ (main frame of Fig. 3 ), using the data corresponding to the case where $\boldsymbol{H}$ is parallel to the $(x, z)$ plane. The $\chi_{0}$ data used for this plot are the susceptibility data of Fig. 1, corrected by the subtraction of the impurity part, as we have described in Ref. 12 . The reason for considering the ratio $F / T$ in Fig. 3 is that the tem-

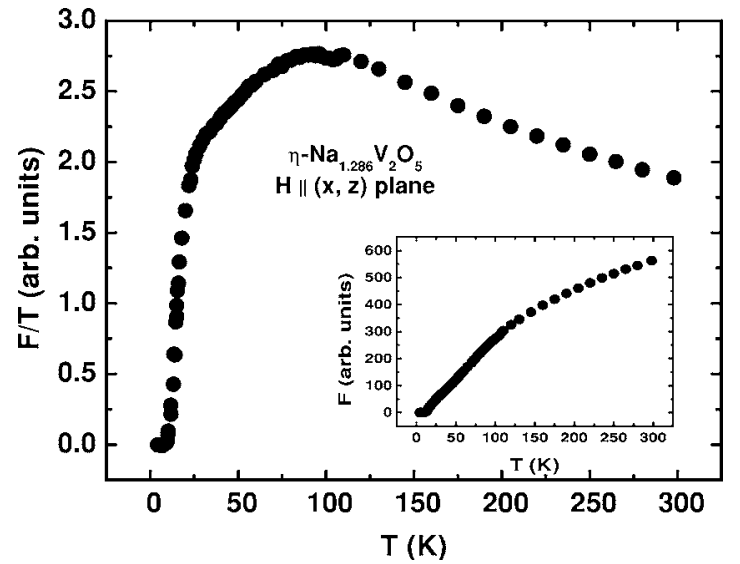

FIG. 3. Temperature dependence of the quantity $F / T$, where $F$ is given by the second part of expression (3), for $\boldsymbol{H}$ parallel to the $(x, z)$ plane of the structure of $\eta-\mathrm{Na}_{1.286} \mathrm{~V}_{2} \mathrm{O}_{5}$. Inset: the temperature dependence of $F$.

perature dependence of this ratio provides much more detail than that of $F$ itself, since the multiplication of $\chi_{0} \Delta H$ by $T$ smears out any eventual features in the curve, particularly at low temperature.

The $F / T$ curve plotted in Fig. 3 shows a broad maximum around $100 \mathrm{~K}$, followed by a rapid decrease towards zero which starts around $17-18 \mathrm{~K}$. Note that $\chi_{0}(T)$, presented in Fig. 1, decreases in this temperature interval much more slowly. Therefore, we conclude that the sharp increase in $\Delta H(T)$ (regime iii) is due to the decrease of $\chi_{0}(T)$ between 60 and $17 \mathrm{~K}$, and that the following decrease of $\Delta H(T)$ below $16 \mathrm{~K}$ (regime iv) arises mainly from the fall of $F / T$. Hence the low- $T$ peak in the linewidth (Fig. 2) simply results from the nonsimultaneous decrease towards zero of $F / T$ and $\chi_{0}$.

It should now be interesting to check, in the case of other spin-gap systems such as $\alpha^{\prime}-\mathrm{NaV}_{2} \mathrm{O}_{5}$ or $\mathrm{CuGeO}_{3}$ which we mentioned above, if the minimum observed at low temperature in the ESR linewidth of these compounds is in fact of magnetic origin or not.

In conclusion, the treatment of the spin dynamics via the memory function formalism has allowed us, by experimentally determining the temperature dependence of the memory function, to provide an explanation for the low-temperature behavior of the ESR linewidth of $\eta-\mathrm{Na}_{1.286} \mathrm{~V}_{2} \mathrm{O}_{5}$. We suggest that the various features observed at low $T$ in the temperature dependence of the linewidth of this compound are simply related to the way in which the magnetic susceptibility and the memory function depend on temperature, rather than to a specific magnetic origin. Such a treatment should pave the way to check the case of many other systems. 
${ }^{1}$ M. Lohmann, H.-A. Krug von Nidda, M. V. Eremin, A. Loidl, G. Obermeier, and S. Horn, Phys. Rev. Lett. 85, 1742 (2000).

${ }^{2}$ R. M. Eremina, M. V. Eremin, V. N. Glazkov, H.-A. Krug von Nidda, and A. Loidl, Phys. Rev. B 68, 014417 (2003).

${ }^{3}$ B. Grenier, P. Monod, M. Hagiwara, M. Matsuda, K. Katsumata, S. Clément, J.-P. Renard, A. L. Barra, G. Dhalenne, and A. Revcolevschi, Phys. Rev. B 65, 094425 (2002).

${ }^{4}$ S. Bertaina, V. A. Pashchenko, A. Stepanov, T. Masuda, and K. Uchinokura, Phys. Rev. Lett. 92, 057203 (2004).

${ }^{5}$ R. Kubo and K. Tomita, J. Phys. Soc. Jpn. 9, 888 (1954).

${ }^{6}$ H. Mori, Prog. Theor. Phys. 34, 423 (1965).

${ }^{7}$ P. M. Richards, Phys. Rev. 142, 189 (1966).

${ }^{8}$ M. Oshikawa and I. Affleck, Phys. Rev. B 65, 134410 (2002).

${ }^{9}$ P. Millet, J.-Y. Henry, and J. Galy, Acta Crystallogr., Sect. C: Cryst. Struct. Commun. 55, 276 (1999).

${ }^{10}$ M. Isobe, Y. Ueda, Y. Oka, and T. Yao, J. Solid State Chem. 145, 361 (1999).

${ }^{11}$ M.-H. Whangbo and H.-J. Koo, Solid State Commun. 115, 115 (2000).
${ }^{12}$ F. Duc, P. Millet, S. Ravy, A. Thiollet, F. Chabre, A. M. Ghorayeb, F. Mila, and A. Stepanov, Phys. Rev. B 69, 094102 (2004).

${ }^{13}$ J. Choukroun, V. A. Pashchenko, Y. Ksari, J. Y. Henry, F. Mila, P. Millet, P. Monod, A. Stepanov, J. Dumas, and R. Buder, Eur. Phys. J. B 14, 655 (2000).

${ }^{14}$ V. A. Pashchenko, A. Sulpice, F. Mila, P. Millet, A. Stepanov, and P. Wyder, Eur. Phys. J. B 21, 473 (2001).

${ }^{15}$ M. S. Seehra and T. G. Castner, Jr., Solid State Commun. 8, 787 (1970).

${ }^{16}$ H. W. de Wijn, L. R. Walker, J. L. Davis, and H. J. Guggenheim, Solid State Commun. 11, 803 (1972).

${ }^{17}$ F. Reynaud, D. Mertz, F. Celestini, J.-M. Debierre, A. M. Ghorayeb, P. Simon, A. Stepanov, J. Voiron, and C. Delmas, Phys. Rev. Lett. 86, 3638 (2001).

${ }^{18}$ S. Taniguchi, Y. Kobayashi, M. Kasai, K. Kodama, and M. Sato, J. Phys. Soc. Jpn. 66, 3660 (1997).

${ }^{19}$ M. J. Hennessy and P. M. Richards, Phys. Rev. B 7, 4084 (1973).

${ }^{20}$ M. Oshikawa and I. Affleck, Phys. Rev. Lett. 82, 5136 (1999). 